\title{
Integration time in space experiments to test the equivalence principle
}

\author{
A. M. Nobili, ${ }^{1,2, *}$ R. Pegna, ${ }^{2}$ M. Shao, ${ }^{3}$ S. G. Turyshev, ${ }^{3}$ G. Catastini, ${ }^{4}$ A. Anselmi, ${ }^{4}$ R. Spero, ${ }^{3}$ S. Doravari, ${ }^{5}$ \\ G. L. Comandi, ${ }^{2}$ D. M. Lucchesi, ${ }^{2,6}$ and A. De Michele ${ }^{1}$ \\ ${ }^{1}$ Department of Physics "E. Fermi," University of Pisa, Largo B. Pontecorvo 3, 56127 Pisa, Italy \\ ${ }^{2}$ INFN-Istituto Nazionale di Fisica Nucleare, Sezione di Pisa, Largo B. Pontecorvo 3, 56127 Pisa, Italy \\ ${ }^{3}$ Jet Propulsion Laboratory, California Institute of Technology, \\ 4800 Oak Grove Drive, Pasadena, California 91109, USA \\ ${ }^{4}$ Thales Alenia Space Italia, Strada Antica di Collegno 253, 10146 Torino, Italy \\ ${ }^{5}$ California Institute of Technology, Pasadena, California 91109, USA \\ ${ }^{6}$ INAF-Istituto Nazionale di Astrofisica, IAPS, Via Fosso del Cavaliere 100, 00133 Roma, Italy \\ (Received 1 March 2013; revised manuscript received 3 November 2013; published 25 February 2014)
}

\begin{abstract}
The integration time required by space experiments to perform high accuracy tests of the universality of free fall and the weak equivalence principle is a crucial issue. It is inversely proportional to the square of the acceleration to be measured, which is extremely small; the duration of the mission is a severe limitation and experiments in space lack repeatability. An exceedingly long integration time can therefore rule out a mission target. We have evaluated the integration time due to thermal noise from gas damping, Johnson noise and eddy currents — which are independent of the signal frequency — and to internal damping, which is known to decrease with increasing frequency. It is found that at low frequencies thermal noise from internal damping dominates. In the "Galileo Galilei" proposed space experiment to test the equivalence principle to $10^{-17}$ the rapid rotation of the satellite $(1 \mathrm{~Hz})$ up-converts the signal to a frequency region where thermal noise from internal damping is lower than gas damping and only a factor 2 higher than Johnson noise, with a total integration time of 2.4 to 3.5 hours even in a very conservative estimate. With an adequate readout and additional care in reducing systematics the test could be improved by another order of magnitude, close to $10^{-18}$, requiring a hundred times longer - still affordable-integration time of 10 to 14.6 days. $\mu$ SCOPE, a similar room temperature mission under construction by the French space agency to be launched in 2015 , aims at a $10^{-15}$ test with an estimated integration time of 1.4 days. Space tests using cold atoms and atom interferometry have been proposed to be performed on the space station (Q-WEP, to $10^{-14}$ ) and on a dedicated mission (STE-QUEST, to $10^{-15}$ like $\mu$ SCOPE). In this case integration is required in order to reduce single shot noise. European Space Agency funded studies report an integration time of several months and a few years respectively.
\end{abstract}

DOI: 10.1103/PhysRevD.89.042005

PACS numbers: 04.80.Cc, 07.87.+v, 62.40.+i

\section{INTRODUCTION}

General relativity is founded on the assumption that in a gravitational field all bodies are equally accelerated [universality of free fall (UFF)]. Tests of UFF, hence of the equivalence between inertial and gravitational mass [weak equivalence principle (WEP)], require us to measure very small accelerations acting between two test masses of different composition freely falling in the gravitational field of a source body, such as Earth (see e.g. [1,2]). No differential acceleration should be detected between the test masses if UFF/WEP holds. The best results to date have been obtained with macroscopic test bodies very weakly coupled in slowly rotating torsion balances, confirming $\mathrm{UFF} / \mathrm{WEP}$ to $\simeq 10^{-13}$ in the field of Earth [3] and to several parts in $10^{13}$ in the field of the Sun [4] (see [5], Table 3).

Corresponding author. nobili@dm.unipi.it
In the gravitational field of Earth much better results can be obtained if the experiment is carried out inside a spacecraft orbiting at low altitude $h$. If the goal is to test UFF/WEP to the level $\eta=\Delta a / g(h)$, a differential acceleration $\Delta a$ must be measured between two test masses of different composition falling in the field of Earth at altitude $h$ with the average acceleration $g(h)$. The corresponding differential force is $F_{\text {signal }}=\mu g(h) \eta, \mu=m_{1} m_{2} /\left(m_{1}+m_{2}\right)$ being the reduced mass of the test bodies of mass $m_{1}, m_{2}$ respectively.

The "Galileo Galilei" (GG) space experiment [1] that we are involved in aims at testing UFF to the level $\eta_{G G}=$ $10^{-17}$ with two concentric coaxial test cylinders of equal mass $\left(m_{1}=m_{2}=10 \mathrm{~kg}, \mu=5 \mathrm{~kg}\right)$. They are very weakly coupled in the plane perpendicular to the symmetry axis so that the natural period of oscillation relative to each other is very long $(\simeq 540 \mathrm{~s})$ yielding high sensitivity to differential forces. They are enclosed in a small spacecraft, also with cylindrical symmetry, to fly in a near circular, Sunsynchronous orbit around Earth at $h \simeq 600 \mathrm{~km}$ altitude. 
The whole spacecraft rotates around the symmetry axis at $1 \mathrm{~Hz}$ rate-provided once for all at the start of the mission-and in so doing it is passively stabilized. The target differential acceleration between the test cylinders is $a_{G G} \simeq 8 \times 10^{-17} \mathrm{~ms}^{-2}$. It gives rise to a displacement of the centers of mass of the test bodies relative to each other pointing to the center of mass of Earth as the satellite orbits around it at frequency $\nu_{\text {orb }} \simeq 1.7 \times 10^{-4} \mathrm{~Hz}$. The size of the target differential displacement is $\simeq 0.6 \mathrm{pm}$. The target differential force is $F_{G G} \simeq 4 \times 10^{-16} \mathrm{~N}$.

Error sources are either systematic or random. Assuming that systematic errors sources can be adequately reduced and that readout noise is not an issue, we investigate random error sources due to thermal noise of various origins at thermal equilibrium. Once the spectral density of all thermal noise sources has been minimized by acting on the physical parameters they depend upon, the only way to bring the effects of random thermal disturbances below the target signal is by increasing the integration time $t_{\text {int }}$ (the duration of the experiment) since the size of the integrated random noise decreases as $1 / \sqrt{t_{\text {int }}}$. However, if the signal to be detected is very small the integration time required can be prohibitively long-especially for an experiment to be carried out in space-which makes a careful analysis of thermal noise and the consequent integration time very important.

\section{THERMAL NOISE AND INTEGRATION TIME}

The fluctuation-dissipation theorem [6] gives a quantitative relationship between dissipation and certain fluctuations. An intuitive interpretation of this fact is given by the authors as follows. A dissipative process involves the interaction of two systems: a dissipative system and a source system. The dissipative system is capable of absorbing energy under the action of a periodic force, and the source system is the one which provides this periodic force and delivers energy to the dissipative system. As long as the two systems are isolated from each other, the source system (e.g. a pendulum) maintains its dynamics (e.g. its periodic motion), and we can say that it possesses an internal coherence. However, once the source system interacts with the dissipative one its energy is sapped away, the internal coherence is destroyed and it is left with only the random disordered energy typical of thermal equilibrium $\left(\simeq k_{B} T\right.$, with $T$ the equilibrium temperature and $k_{B}$ the Boltzmann constant). Thus, random fluctuations generated in the dissipative system and acting on the source system give rise to its loss of coherence. As stated in [6], dissipation is the macroscopic manifestation of the disordering effect of the Nyquist fluctuations [7] and, as such, it is necessarily quantitatively correlated with the fluctuations.

The power spectral density (PSD) of the random force associated with some dissipation expressed by a damping coefficient $\gamma$ at equilibrium temperature $T$ is given (using the "hat" symbol for the Fourier transform) by

$$
\left\langle\left|\hat{F}_{\text {th }}(\omega)\right|^{2}\right\rangle=4 k_{B} T \gamma \text {. }
$$

The dissipative phenomena which give rise to a thermal noise random force can be of various kinds. For a given target force $F\left(\omega_{\text {signal }}\right)$ acting at frequency $\omega_{\text {signal }}$ the integration time required for the measured force signal to emerge above the competing thermal noise force by the factor signal-to-noise ratio (SNR) is

$$
t_{\text {int }}=\mathrm{SNR}^{2} \cdot \frac{\left\langle\left|\hat{F}_{\text {th }}\left(\omega_{\text {signal }}\right)\right|^{2}\right\rangle_{\text {tot }}}{F\left(\omega_{\text {signal }}\right)^{2}}
$$

where

$$
\left\langle\left|\hat{F}_{\text {th }}\left(\omega_{\text {signal }}\right)\right|^{2}\right\rangle_{\text {tot }}=\sum_{i}\left\langle\left|\hat{F}_{\text {th }}\left(\omega_{\text {signal }}\right)_{i}\right|^{2}\right\rangle
$$

and the index $i$ refers to the $i$ th kind of dissipative phenomenon which gives rise to a thermal noise fluctuating force. Thus, aiming at a 10 times better sensitivity would require, with the same limiting level of thermal noise, a 100 times longer integration time.

UFF/WEP tests are concerned about comparing gravitational and inertial forces (i.e. Earth's attraction and the centrifugal force while orbiting around it) which are proportional to the gravitational and inertial mass respectively, in the search for a deviation of their ratio from +1 . Therefore, the target signal is a differential acceleration while thermal noise forces are nongravitational forces producing smaller accelerations if the mass of the body is larger. More massive test bodies should therefore be used whenever possible.

\section{THE CASE OF SPACE EXPERIMENTS TO TEST THE EQUIVALENCE PRINCIPLE}

Experiments to test UFF/WEP belong to the category of extremely small force gravitational experiments, and hence require great care in dealing with thermal noise. We analyze three types of thermal noise forces which physicists have faced-and still face-in such small force experiments. They are due to gas damping in the presence of residual air surrounding the test masses; Johnson noise and eddy currents in the presence of a magnetic field; and internal damping due to losses in the suspensions of the test bodies.

\section{A. Thermal noise due to gas damping}

Let us first consider gas damping. In the small force experiments that we are interested in, the residual pressure of the gas surrounding the test mass is low and the mean free path of the gas molecules is longer than the size of the test mass. In these conditions the gas damping coefficient $\gamma_{\mathrm{gas}}$ can be written as (see e.g. [8])

$$
\gamma_{\mathrm{gas}}=\frac{p}{v_{T}} \cdot \mathcal{A}
$$


where $p$ is the gas pressure, $v_{T}=\sqrt{k_{B} T / m_{\mathrm{o}}}$ is the thermal velocity of the gas molecules of mass $m_{\mathrm{o}}$ at equilibrium temperature $T$ in one direction and $\mathcal{A}$ is the surface cross section of the test mass depending on its specific geometry. The gas damping coefficient does not depend on the frequency at which the test mass is oscillating.

In the simplified assumption that the test body is surrounded by an "infinite" volume of gas, in which case multiple bounces typical of small gaps do not occur, the cross section $\mathcal{A}_{\infty}$ (the subscript refers to the assumption made) has been computed by the authors of Ref. [8] for various geometrical shapes of the body. For a solid cylinder of radius $R$ and height $H$ sensitive to forces acting in the plane perpendicular to the symmetry axis they find that the translation damping coefficient is proportional to the geometrical cross section:

$$
\mathcal{A}_{\infty}=\pi R^{2} \sqrt{\frac{2}{\pi}}\left[1+\frac{3 H}{2 R}\left(1+\frac{\pi}{6}\right)\right] .
$$

If the test mass is not surrounded by an "infinite" volume of gas because of small gaps (compared to its size) between its surfaces and the surrounding enclosure, the effective cross section increases due to multiple bounces of the gas molecules.

For cubic test masses completely surrounded by a nearby enclosure the amplification factor has been estimated numerically with the aid of some experimental checks [9]. A plot is reported which provides the simulated amplification factor as function of the ratio between the size $d$ of the gap (between the cube and the enclosure surrounding it on all sides) and the length $s$ of the side of the cube for $0.005<d / s<50$ ([9], Fig. 5). The authors give also an analytic expression for the amplification factor which however is valid only for $d / s \ll 0.01$.

In the current GG baseline the inner test cylinder is made of Ti $(5.55 \mathrm{~cm}$ inner radius, $8.51 \mathrm{~cm}$ outer radius, $17 \mathrm{~cm}$ height) and the outer cylinder is made of $\mathrm{Be}(10.51 \mathrm{~cm}$ inner radius, $13.06 \mathrm{~cm}$ outer radius, $28.63 \mathrm{~cm}$ height). They are separated from each other by a $2 \mathrm{~cm}$ gap, and the inner one is separated from the shaft by a similar gap. To the contrary, on both sides in the direction of the symmetry axis, as well as outside of the outer cylinder, the volume available to the gas is much larger than the corresponding size of the bodies and can be regarded as "infinite." For the cylindrical surfaces characterized by a gap from the nearby cylindrical surface smaller than the radius of the cylinder we estimate the amplification factor from the simulation reported by [9] in Fig. 5 (translational case) for a cube with a similar gap on all faces and side length equal to the radius of the cylinder.

Gas molecules trapped in between the GG test cylinders and in between the shaft and the inner cylinder have the chance to access large volumes at the two ends of the symmetry axis, thus reducing the number of multiple bounces on those surfaces. Therefore, an estimate of the amplification factors based as in [9] on the case of a test mass completely surrounded by a nearby enclosure with a small gap is a worst case estimate for GG.

Within this conservative estimate the amplification factors turn out to be 4 and 2 for the inner cylinder (for its outer and inner surfaces respectively) and 5 for the inner surface of the outer cylinder, taking also into account that it faces the outer surface of the inner cylinder with a $2 \mathrm{~cm}$ gap only for the height of the inner cylinder while in the remaining the ratio $d / s$ is close to 1 , yielding no amplification. We evaluate the gas damping coefficients at $T \simeq 300 \mathrm{~K}$ equilibrium temperature and $p \simeq 10^{-5} \mathrm{~Pa}$ pressure from the residual gas whose molecules have $m_{\mathrm{o}} \simeq 5 \times 10^{-26} \mathrm{~kg}$. We compute the differential component of the gas damping force on each of the four surfaces of the test cylinders and finally derive the PSD of the total differential force due to gas damping in the assumption that they are all random. Note that a force acting only on one of the two test bodies, say $m_{1}$, results in a differential force between the two a fraction $m_{2} /\left(m_{1}+m_{2}\right)$ smaller. If $m_{1}=m_{2}$ the differential force is a factor 2 smaller, yielding - in the case of a damping force-a damping coefficient a factor 4 smaller. We get

$$
\left\langle\left|\hat{F}_{\text {th-gas }}(\omega)\right|^{2}\right\rangle \simeq 2.2 \times 10^{-28} \mathrm{~N}^{2} / \mathrm{Hz}
$$

corresponding to a gas damping coefficient

$$
\gamma_{\text {gas }} \simeq 1.3 \times 10^{-8} \mathrm{~kg} / \mathrm{s} .
$$

In the same conservative assumptions, but allowing for the gas damping forces in the gap between the concentric cylinders (i.e. the force on the outer surface of the inner cylinder and the force on the inner surface of the outer one) to be correlated to each other, we would obtain a slightly larger effect of $\simeq 3.8 \times 10^{-28} \mathrm{~N}^{2} / \mathrm{Hz}$ and a correspondingly larger damping coefficient of $\simeq 2.3 \times 10^{-8} \mathrm{~kg} / \mathrm{s}$.

\section{B. Johnson noise and eddy currents}

A magnetic shield is required in order to attenuate the disturbances caused by Earth's magnetic field $B_{\oplus} \simeq 5 \times$ $10^{-7} \mathrm{~T}$ on the test masses at the same frequency as the target violation signal or at frequencies very close to it. In GG a 150 attenuation factor is sufficient, to be achieved with a $\mu$-metal shield which can be located on the inner surface of the spacecraft.

The residual magnetic field inside the volume surrounded by a $\mu$-metal shield is known to exhibit spatial gradients even if the magnetic field outside it is uniform. Gradients inside the shield are mitigated if the shield has almost uniform thickness and magnetic permeability and if it is close to spherically symmetric, being zero in the ideal case that the shield is spherical, it has uniform thickness and uniform permeability. 
In the following we shall make the worst case assumption that the gradient inside the shield is as large as $B / r_{s}$, where $B=B_{\oplus} / 150 \simeq 3.33 \times 10^{-7} \mathrm{~T}$ is the magnetic field of Earth attenuated by the shield and $r_{s} \simeq 0.7 \mathrm{~m}$ is the average radius of the shield. In the case of GG the shape of the shield is essentially the shape of the spacecraft body, which is close to having spherical symmetry. Moreover, its rapid rotation helps to further reduce effects due to imperfections.

If the test masses are conductors with resistance $\mathcal{R}$ at temperature $T$, Johnson noise inside them gives rise to fluctuating current loops, hence to magnetic moments which-in the presence of a nonzero gradient of the magnetic field-give rise to a fluctuating force competing with the target violation signal. The power spectral density of the Johnson voltage noise is known to be

$$
\left\langle\left|\hat{V}_{J}(\omega)\right|^{2}\right\rangle=4 k_{B} T \mathcal{R}
$$

from which, by Ohm's law, the PSD of the fluctuating currents noise is derived:

$$
\left\langle\left|\hat{I}_{J}(\omega)\right|^{2}\right\rangle=\frac{\left\langle\left|\hat{V}_{J}(\omega)\right|^{2}\right\rangle}{\mathcal{R}^{2}}=\frac{4 k_{B} T}{\mathcal{R}}
$$

giving rise to the magnetic moment noise,

$$
\left\langle\left|\hat{m}_{J}(\omega)\right|^{2}\right\rangle \lesssim\left\langle\left|\hat{I}_{J}(\omega)\right|^{2}\right\rangle H^{4} \lesssim \frac{4 K_{B} T}{\mathcal{R}} H^{4}
$$

where $H^{2}$ ( $H$ being the height of a test cylinder) is an upper bound for the area of the current loops.

With a magnetic field gradient $B / r_{s}$ inside the spacecraft the PSD of the resulting fluctuating force on the test cylinder is

$$
\left\langle\left|\hat{F}_{\mathrm{th}-J}(\omega)\right|^{2}\right\rangle \lesssim\left\langle\left|\hat{m}_{J}(\omega)\right|^{2}\right\rangle \frac{B^{2}}{r_{s}^{2}} \lesssim \frac{4 K_{B} T}{\mathcal{R}} \frac{B^{2} H^{4}}{r_{s}^{2}}
$$

As a rough estimate of the resistance $\mathcal{R}$ for a hollow test cylinder we take

$$
\mathcal{R} \simeq \frac{\rho H}{\pi\left(r_{2}^{2}-r_{1}^{2}\right)}
$$

where $\rho=1 / \sigma$ is the resistivity of the material $\left(\rho_{\mathrm{Be}} \simeq 4 \times 10^{-8} \Omega \mathrm{m}\right.$ for the outer cylinder and $\rho_{\mathrm{Ti}} \simeq 4.2 \times$ $10^{-7} \Omega \mathrm{m}$ for the inner one) and $r_{1}, r_{2}$ are the inner and outer radii respectively of the cylinder of height $H$ (as given in Sec. III A). The largest effect is on the Be cylinder, because of its lower resistivity and bigger height. We therefore take as an upper bound of the differential effect between the test masses the total effect on the Be cylinder (at $T \simeq 300 \mathrm{~K}$ ):

$$
\left\langle\left|\hat{F}_{\text {th }-J}(\omega)\right|^{2}\right\rangle \lesssim 4.15 \times 10^{-29} \mathrm{~N}^{2} / \mathrm{Hz}
$$

which is about a factor 5 smaller than the value (6) due to gas damping. A more detailed estimate taking into account symmetry and rapid rotation in reducing the magnetic field gradient would yield an even smaller effect.

By writing (11) as

$$
\left\langle\left|\hat{F}_{\mathrm{th}-J}(\omega)\right|^{2}\right\rangle=4 k_{B} T \gamma_{J}
$$

we obtain the damping coefficient associated with Johnson noise:

$$
\gamma_{J} \lesssim \frac{H^{4}}{r_{s}^{2}} \frac{B^{2}}{\mathcal{R}}
$$

Its value corresponding to (13) is

$$
\gamma_{J} \lesssim 2.5 \times 10^{-9} \mathrm{~kg} / \mathrm{s}
$$

In $\mathrm{GG}$ the test cylinders are weakly coupled in the plane perpendicular to the spin/symmetry axis with a natural frequency of oscillation relative to each other $\omega_{n} \simeq 2 \pi / 540 \mathrm{rads}^{-1}$. According to industrial studies of the GG experiment in space, based on realistic simulations and measurement errors, the largest relative displacement between the centers of mass in the sensitive plane is $\Delta r_{\text {max }} \simeq 10^{-8} \mathrm{~m}$ (see [10], Ch. 6, and [11]). If such motion occurs in the presence of a nonzero gradient of the magnetic field of Earth eddy currents are generated inside the test cylinders. An upper bound to the PSD of these currents is

$$
\left\langle\left|\hat{I}_{\text {eddy }}(\omega)\right|^{2}\right\rangle \lesssim \frac{B^{2} H^{4} \omega_{n}^{2} \Delta r_{\max }^{2}}{\mathcal{R}^{2} r_{s}^{2}}
$$

By comparison with the Johnson noise currents (9) we have the ratio

$$
\frac{\left\langle\left|\hat{I}_{J}(\omega)\right|\right\rangle}{\left\langle\left|\hat{I}_{\text {eddy }}(\omega)\right|\right\rangle} \gtrsim \sqrt{4 k_{B} T \frac{\mathcal{R} r_{s}^{2}}{B^{2} H^{4} \omega_{n}^{2} \Delta r_{\max }^{2}}}
$$

showing that, as far as the gradients of the magnetic field are concerned, Johnson noise dominates by far over eddy currents noise [the ratio (18) is $\gtrsim 2.2 \times 10^{4}$ for the Be test cylinder].

No eddy currents are generated in a conductor immersed in a uniform and constant magnetic field as long as it moves with pure translational motion, because there is no change in the flux of the magnetic field in a loop inside the conductor.

If the conductor rotates and the constant magnetic field has a nonzero component transverse to its rotation axis there is a changing flux and there are eddy currents which dissipate rotation energy. The power dissipated by eddy currents in slowly rotating hollow cylinders has been calculated by [12] for various geometrical dimensions of the cylinders. They give [[12], Eq. (10)] $P=\pi \sigma \omega_{\text {spin }}^{2} B^{2} r_{2}^{5} f(\lambda, \mu)$, where $\sigma$ is the conductivity of the cylinder, $r_{2}$ its external radius and the 
function $f(\lambda, \mu)$ is a numerical factor depending on the values $\lambda, \mu$ of the inner radius and semiheight of the cylinder respectively expressed in units of its external radius. In GG the largest effect is on the Be test cylinder for which, with the numerical values reported in Sec. III A, it is $f_{\mathrm{Be}}\left(\lambda_{\mathrm{Be}}, \mu_{\mathrm{Be}}\right) \simeq 0.095$ (see Fig. 2 in [12]), yielding $P_{\mathrm{Be}} \simeq 1.24 \times 10^{-9} \mathrm{~W}$. With $1 \mathrm{~s}$ spin period, this is also the energy dissipated by eddy currents per spin period. Knowing the rotation energy of the test cylinder $\left(E_{\mathrm{Be}}=0.5 I_{\mathrm{Be}} \omega_{\text {spin }}^{2} \simeq 2.8 \mathrm{~J}\right.$, with $I_{\mathrm{Be}}=0.14 \mathrm{~kg} \mathrm{~m}^{2}$ the moment of inertia with respect to the rotation/symmetry axis) we get the quality factor corresponding to these losses: $Q_{\mathrm{Be}} \simeq 2 \pi \cdot E_{\mathrm{Be}} /\left(1.24 \times 10^{-9} \mathrm{~J}\right) \simeq 1.4 \times 10^{10}$. As long as the magnetic field is uniform the power dissipated in the rotating test cylinders has no effect on the translational motion of their centers of mass, and hence on the equivalence principle violation signal being sought.

\section{Thermal noise due to internal damping}

We have seen that the contribution to thermal noise from both gas damping and Johnson noise is frequency independent. Whatever the frequency of the signal, their contribution to the total thermal noise competing with it will not change.

To the contrary, the frequency of the signal is relevant when we consider thermal noise due to internal damping (also referred to as structural or material damping) in which case dissipation occurs inside the weak mechanical joints which suspend (or couple, in the case of GG) the test masses. In this case the damping coefficient $\gamma$ in (1) is known to depend on the frequency $\omega$ in the form [see [13], Sec. V, Eq. (14)]

$$
\gamma_{\mathrm{id}}(\omega) \simeq \frac{k \phi(\omega)}{\omega}=\frac{\mu \omega_{n}^{2} \phi(\omega)}{\omega}
$$

where $k$ is the elastic constant of the mechanical suspension and $\phi(\omega)$ its loss angle at the oscillation frequency $\omega$. In this form it has been confirmed by slowly rotating torsion balances testing the equivalence principle on ground up to their maximum rotation frequency of about $1 \mathrm{mHz}$, slightly below the resonance, while above it the autocollimator noise dominates, as the authors report ([14], Fig. 20). In the case of GG $k$ is the elastic constant of the 2D laminar joints which couple the concentric test cylinders in each direction of the sensitive plane perpendicular to the axis of symmetry; hence, $k=\mu \omega_{n}^{2}$ with $\mu=5 \mathrm{~kg}$ the reduced mass of the two cylinders and $\omega_{n}=2 \pi / 540 \mathrm{rad} \mathrm{s}^{-1}$ the frequency of natural oscillation of the cylinders relative to each other.

It is apparent from (19) that if the loss angle is independent of frequency the internal damping coefficient is lower at higher frequency, yielding a shorter integration time. Indeed, there is experimental evidence that the loss angle too decreases at higher frequency (see e.g. [15]) making the decrease of internal damping at higher frequency even stronger.

Thermal noise from internal damping is known to be a serious issue in small force experiments where the target signal has low frequency.

If UFF/WEP is tested in a spacecraft that does not rotate with respect to inertial space while orbiting Earth at low altitude, in an inertial reference frame centered on the center of mass of Earth a violation signal would have the (low) orbital frequency $\left(\omega_{\text {orb }} \simeq 2 \pi \cdot 1.7 \times 10^{-4} \mathrm{rad} \mathrm{s}^{-1}\right)$. In GG, by rotating around the symmetry axis the whole spacecraft which encloses-in a nested configurationthe test cylinders and their readout, the spacecraft is passively stabilized and, at the same time, the signal is up-converted to the rotation frequency $\omega_{\text {spin }} \simeq 2 \pi \mathrm{rad} \mathrm{s}^{-1}$ at which the satellite is stabilized. The main features of the GG sensor are sensitivity in 2D, rotation frequency higher than the natural frequency of the mechanical oscillator (by the factor 540), and up-conversion of the signal frequency to the much higher rotation frequency (by a factor almost 5900). It has been demonstrated [16] that in these conditions the relevant coefficient of internal damping is

$$
\gamma_{\text {id }}\left(\omega_{\text {spin }}\right) \simeq \frac{\mu \omega_{n}^{2} \phi\left(\omega_{\text {spin }}\right)}{\omega_{\text {spin }}} \simeq 5.4 \times 10^{-9} \mathrm{~kg} / \mathrm{s}
$$

[at $\omega_{\text {spin }} \simeq 2 \pi \mathrm{rad} \mathrm{s}^{-1}, \phi\left(\omega_{\text {spin }}\right) \simeq 5 \times 10^{-5}$ ] yielding for the PSD of the corresponding thermal noise force competing with the signal up-converted to $\omega_{\text {spin }}$ the value

$$
\left\langle\left|\hat{F}_{\text {th-id }}\left(\omega_{\text {spin }}\right)\right|^{2}\right\rangle \simeq 4 k_{B} T \gamma_{\text {id }}\left(\omega_{\text {spin }}\right) \simeq 8.9 \times 10^{-29} \mathrm{~N}^{2} / \mathrm{Hz} .
$$

In the absence of rotation, the PSD of the thermal noise force competing with a violation signal at the orbital frequency $\omega_{\text {orb }} \simeq 2 \pi \cdot 1.7 \times 10^{-4} \mathrm{rad} \mathrm{s}^{-1}$ would be

$$
\left\langle\left|\hat{F}_{\text {th-id }}\left(\omega_{\text {orb }}\right)\right|^{2}\right\rangle=\frac{4 k_{B} T \mu \omega_{n}^{2} \phi\left(\omega_{\text {orb }}\right)}{\omega_{\text {orb }}}
$$

and hence

$$
\left\langle\left|\hat{F}_{\text {th-id }}\left(\omega_{\text {orb }}\right)\right|^{2}\right\rangle \gtrsim 5.3 \times 10^{-25} \mathrm{~N}^{2} / \mathrm{Hz},
$$

where we have used the symbol $\gtrsim$ instead of $\simeq$ becausefor the same suspension - the value of the loss angle is known to depend to some extent on the frequency at which it oscillates, being higher at lower frequencies. Thus, with

$$
\frac{\left\langle\left|\hat{F}_{\text {th-id }}\left(\omega_{\text {orb }}\right)\right|^{2}\right\rangle}{\left\langle\left|\hat{F}_{\text {th-id }}\left(\omega_{\text {spin }}\right)\right|^{2}\right\rangle} \gtrsim \frac{\omega_{\text {spin }}}{\omega_{\text {orb }}} \simeq 5900
$$

thermal noise from internal damping for the signal at orbital frequency would dominate by far and make the integration 
time (2) longer by the same factor. This fact shows well the advantage of GG in modulating the signal by rapid rotation.

Operating the experiment at low temperature ( $\simeq 2 \mathrm{~K}$ instead of $\simeq 300 \mathrm{~K})$ would have the advantage of reducing all sources of thermal noise alike. As far as internal damping is concerned a reduction of the integration time by the factor $\simeq 150$ in a cryogenic experiment would be less effective than up-converting the signal to high frequency [see (24)] except for the cases in which a reduction of the loss angle is achieved at low temperature.

A drastic reduction of thermal noise would not help very much if the response of the sensor to the signal were attenuated due to its up-conversion above the resonance (or natural) frequency of the oscillator. This fact is well known to occur in $1 \mathrm{D}$ mechanical oscillators where the force measured is smaller than the force applied if it is applied at frequency $\omega$ higher than the resonance frequency of the oscillator $\omega_{n}$, with an attenuation factor $\omega^{2} / \omega_{n}^{2}$ for frequencies much higher than the resonance (see e.g. [17], Vol. 1, Ch. 23). It is also known that no such attenuation occurs if the oscillator has 2 degrees of freedom like GG [see [16], Eq. (20), and [1], Fig. 2]. As shown in Sec. I, the acceleration to be measured for GG to test UFF/WEP to $\eta_{\mathrm{GG}}=10^{-17}$ is $a_{\mathrm{GG}} \simeq 8 \times 10^{-17} \mathrm{~ms}^{-2}$; the same value must be measured when the signal is up-converted by rotation to $\omega_{\text {spin }} \simeq 2 \pi \mathrm{rad} \mathrm{s}^{-1}$.

\section{Integration time}

The square of the force $F_{G G}$ given in Sec. I (the target differential force to be measured for GG to perform a weak equivalence principle test to $\eta_{G G}=10^{-17}$ ) enters at the denominator of (2) for the calculation of the integration time, while at the numerator, assuming all thermal noise sources to be uncorrelated, we have

$$
\begin{aligned}
\left\langle\left|\hat{F}_{\text {th }}\left(\omega_{\text {spin }}\right)\right|^{2}\right\rangle_{\text {tot }}= & \left\langle\left|\hat{F}_{\text {th }- \text { gas }}\right|^{2}\right\rangle+\left\langle\left|\hat{F}_{\text {th-id }}\left(\omega_{\text {spin }}\right)\right|^{2}\right\rangle+\left\langle\left|\hat{F}_{\text {th }-J}\right|^{2}\right\rangle \\
\simeq & 2.2 \times 10^{-28}+8.9 \times 10^{-29} \\
& +4.2 \times 10^{-29} \mathrm{~N}^{2} / \mathrm{Hz} \\
\simeq & 3.5 \times 10^{-28} \mathrm{~N}^{2} / \mathrm{Hz}
\end{aligned}
$$

yielding, for a signal-to-noise ratio of 2, an integration time of about $2.4 \mathrm{~h}$.

If the gas damping thermal noise forces in the gap are correlated we get $\left\langle\left|\hat{F}_{\text {th }}\left(\omega_{\text {spin }}\right)\right|^{2}\right\rangle_{\text {tot }} \simeq 5 \times 10^{-28}$ and $3.5 \mathrm{~h}$ integration time.

We note from (23) that if the frequency of the target signal had not been up-converted to the rapid spin frequency of GG, thermal noise from internal damping would dominate by far making gas damping and Johnson noise irrelevant and requiring, for the same signal and the same signal-to-noise ratio, an integration time of $152 \mathrm{~d}$.

\section{CONCLUSIONS}

Tests of the universality of free fall and the weak equivalence principle in low Earth orbit require us to measure the effect of a very small acceleration at low frequency, typically up-converted to higher frequency for electronic and mechanical noise reduction. Assuming that at this frequency readout noise is adequate to the measurement, and that systematic errors can be reduced below the signal, the experiment is ultimately limited by thermal (random) noise. The integration time required for it to be reduced below the signal is inversely proportional to the square of the force signal to be measured. If the target force is very small, this may be prohibitively long for an experiment to be performed in space.

GG aims at testing the universality of free fall and the weak equivalence principle to $\eta_{G G}=10^{-17}$ (4 orders of magnitude improvement). We have estimated thermal noise due to residual-gas damping, eddy currents and internal damping in the mechanical suspensions finding overallunder conservative assumptions - an integration time of 2.4 to 3.5 hours for a signal-to-noise ratio of 2. Crucial to this result is the up-conversion of the signal to $1 \mathrm{~Hz}$, since it reduces the contribution of internal damping to thermal noise at the frequency that the signal has been up-converted to. The fact that thermal noise results in such a short integration time leaves room for a mission outcome even better than planned. If care is devoted to reducing readout noise and to reducing/separating the most dangerous systematic errors, one further order of magnitude improvement (to $10^{-18}$ ) would require an integration time from 10 to 14.6 days. With no up-conversion, or up-conversion to a low frequency, thermal noise from internal damping would dominate by far and even the current $10^{-17}$ target would require 152 days of integration and therefore would be hard to achieve.

The $\mu$ SCOPE mission, currently under construction and planned for launch in 2015, aims at a space test of UFF/WEP too. With a different sensor design, and upconversion of the signal from the orbital frequency $\nu_{\text {orb- } \mu \text { scope }} \simeq 1.6 \times 10^{-4} \mathrm{~Hz}$ to $\nu_{\text {signal }-\mu \text { scope }} \simeq 7.7 \times 10^{-4} \mathrm{~Hz}$ $\left(\nu_{\text {spin }-\mu \text { scope }}=\nu_{\text {signal }-\mu \text { scope }}-\nu_{\text {orb- } \mu \text { scope }} \simeq 6.1 \times 10^{-4} \mathrm{~Hz}\right)$ the target is set at $\eta_{\mu \text { scope }}=10^{-15}$. The authors report that the dominant source of thermal noise is due to internal damping in the gold wire connecting each test mass to its enclosure, and estimate for the spectral density of the acceleration noise the value $1.4 \times 10^{-12} \mathrm{~ms}^{-2} / \sqrt{\mathrm{Hz}}$ ([18], Table 5; confirmed in [19], p. 7). For a target acceleration signal $a_{\mu \text { scope }} \simeq$ $8 \times 10^{-15} \mathrm{~ms}^{-2}$ and a signal-to-noise ratio $\mathrm{SNR}=2$ the required integration time is $t_{\text {int }-\mu \text { scope }} \simeq 1.23 \times 10^{5} \mathrm{~s}=1.4 \mathrm{~d}$.

Upon solicitation from the European Space Agency (ESA) [20] two studies have been carried out for an atom interferometry test of the weak equivalence principle in space (referred to as Q-WEP) to be performed on the International Space Station [21]. The target was set to 
$10^{-14}$, with the isotopes ${ }^{85} \mathrm{Rb},{ }^{87} \mathrm{Rb}$ and an integration time of several months, for the single shot noise to be reduced below the target signal through a very large number of runs (assuming random runs).

A UFF/WEP test to almost the same $10^{-15}$ target as in $\mu \mathrm{SCOPE}$ is being considered using cold atoms (also ${ }^{85} \mathrm{Rb},{ }^{87} \mathrm{Rb}$ ) and atom interferometry with the dedicated space mission STE-QUEST under study by ESA [22]. In this case an integration time of a few years is needed, comparable to the duration of the mission. This is undoubtedly a weakness because the measurement could either indicate a major new physical result (violation) or simply report detection of a tiny, known, classical effect. With one or just a few measurements available during the entire mission it would be very hard to establish which is the case, making the scientific result of the mission uncertain.
We conclude that the integration time required in space missions to test the weak equivalence principle is a crucial issue worth a careful investigation by the proposing scientists. Its consequences on the mission feasibility, cost and scientific outcome are so far reaching that it should be seriously taken into account by space agencies in evaluating and comparing mission proposals.

\section{ACKNOWLEDGMENTS}

This work has been supported by ASI (Agenzia Spaziale Italiana) and INFN and it was performed in part at JPL, Caltech under a contract with NASA. The contribution of one referee on the effects of eddy currents is gratefully acknowledged.
[1] A. M. Nobili, M. Shao, R. Pegna, G. Zavattini, S. G. Turyshev, D. M. Lucchesi, A. De Michele, S. Doravari, G. L. Comandi, T. R. Saravanan, F. Palmonari, G. Catastini, and A. Anselmi, Classical Quantum Gravity 29, 184011 (2012).

[2] A. M. Nobili, D. M. Lucchesi, M. T. Crosta, M. Shao, S. G. Turyshev, R. Peron, G. Catastini, A. Anselmi, and G. Zavattini, Am. J. Phys. 81, 527 (2013).

[3] S. Schlamminger, K.-Y. Choi, T. A. Wagner, J. H. Gundlach, and E. G. Adelberger, Phys. Rev. Lett. 100, 041101 (2008).

[4] S. Baeßler, B. R. Heckel, E. G. Adelberger, J. H. Gundlach, U. Schmidt, and H. E. Swanson, Phys. Rev. Lett. 83, 3585 (1999).

[5] T. D. Wagner, S. Shlamminger, J. H. Gundlach, and E. G. Adelberger, Classical Quantum Gravity 29, 184002 (2012).

[6] H. B. Callen and T. A. Welton, Phys. Rev. 83, 34 (1951).

[7] H. Nyquist, Phys. Rev. 32, 110 (1928).

[8] A. Cavalleri, G. Ciani, R. Dolesi, M. Hueller, D. Nicolodi, D. Tombolato, S. Vitale, P. J. Wass, and W. J. Weber, Phys. Lett. A 374, 3365 (2010).

[9] A. Cavalleri, G. Ciani, R. Dolesi, A. Heptonstall, M. Hueller, D. Nicolodi, S. Rowan, D. Tombolato, S. Vitale, P. J. Wass, and W. J. Weber, Phys. Rev. Lett. 103, 140601 (2009).

[10] ASI, GG Phase A Study Report, 1998, http://eotvos.dm .unipi.it/ggweb/phaseA.
[11] ASI, GG Phase A-2 Study Report, 2009, http://eotvos.dm .unipi.it/PA2/GGPA2.pdf.

[12] R. Shäfer and C. Heiden, Applied Physics (Berlin) 9, 121 (1976).

[13] P. R. Saulson, Phys. Rev. D 42, 2437 (1990).

[14] E. G. Adelberger, J. H. Gundlach, B. R. Heckel, S. Hoedl, and S. Schlamminger, Prog. Part. Nucl. Phys. 62, 102 (2009).

[15] G. Cagnoli, L. Gammaitoni, J. Kovalik, F. Marchesoni, and M. Punturo, Phys. Lett. A 255, 230 (1999).

[16] R. Pegna, A. M. Nobili, M. Shao, S. G. Turyshev, G. Catastini, A. Anselmi, R. Spero, S. Doravari, G. L. Comandi, and A. De Michele, Phys. Rev. Lett. 107, 200801 (2011).

[17] R. P. Feynman, R. B. Leighton, and M. Sands, The Feynman Lectures on Physics (Addison-Wesley Pub. Co., Reading, MA, 1967).

[18] P. Touboul, Space Sci. Rev. 148, 455 (2009).

[19] P. Touboul, G. Metris, V. Lebat, and A. Robert, Classical Quantum Gravity 29, 184010 (2012).

[20] ESA Invitation to Tender AO/1-6763/11/NL/AF, 2011.

[21] Q-WEP science team, "Q-WEP: Atom Interferometry Test of the Weak Equivalence Principle in Space" ESA (to be published).

[22] STE-QUEST science requirements document, 2012, http:// sci.esa.int/ste-quest. 